\title{
Non-linear Dynamics of Heart Rate Variability in Athletes: Effect of Training
}

\author{
AE Aubert, F Beckers, B Seps \\ Laboratory Experimental Cardiology, University Hospital Gasthuisberg, K. U. Leuven, Leuven, \\ Belgium
}

\begin{abstract}
Only few studies on heart rate variability (HRV) in athletes have been performed so far. Moreover most of the data were obtained from time or frequency analysis. The aim of the present work was to use methods of nonlinear dynamics to study heart rate variability in a young population consisting of athletes and a sedentary group, used as controls.

ECG episodes lasting $10 \mathrm{~min}$ were recorded digitally in supine position in a group of 34 young subjects. Spectral analysis was performed with Fast Fourier transform (FFT). Following non-linear methods were computed: fractal dimension), $1 / f$ slope, approximate entropy (ApEn) and Lyapunov exponent.

Major difference between controls and aerobic athletes was in resting heart rate. ApEn correlated well with power spectral density. Aerobic athletes showed a difference with controls in ApEn and Lyapunov exponent.

Results indicate a possible relation of non-linear indices and autonomic regulation of cardiovascular function.
\end{abstract}

\section{Introduction}

Heart rate variability (HRV) has become a universal tool to study the neural control of the heart i.e. the delicate interaction between sympathetic and vagal influences on heart rate [1] in health and disease. A variety of linear, non-linear, periodical and nonperiodical oscillation patterns are present in heart rate fluctuations [2].

HRV can be quantified by the simple calculation of the mean and standard deviation of RR-intervals in the time domain. Furthermore, in the frequency domain, spectral analysis of HRV reveals two distinct frequency regions in the modulation of heart rate in humans. A high frequency region (0.16-0.4 Hz) which is a marker of vagal modulation, and a low frequency region $(0.04-0.15 \mathrm{~Hz})$, which reflects predominantly sympathetic tone [3] and baroreflex activity [4]. Both time domain and frequency domain methods are based on the assumption that HRV signals are linear [5], and thus these methods cannot fully quantify the dynamical structure of the signal. To assess the non-linear properties, several methods have been proposed in the past: fractal dimension [6], Lyapunov exponents [7], correlation dimension [8,9], 1/f slope [10], approximate entropy [11] and detrended fluctuation analysis [12]. All these methods quantify some non-linear characteristic of HRV. The non-linear methods represent potentially promising tools for heart rate variability assessment, especially in combination with the well known time and frequency domain methods.

The aim of this work was to study heart rate variability (HRV) in a young population with the above mentioned methods and compare nonlinear indices with spectral analysis and to compare indices in differently athletic trained groups.

\section{Methods}

\subsection{Study population}

Thirty four young subjects (all male, 18-34 years of age) were included in this study. All subjects were physically healthy with no history of cardiac disease. The population consisted of 4 groups: 10 endurance trained athletes (aerobic, all mid-distance runners), 7 static trained athletes (anaerobic, high jumpers and javelin throwers), 7 rugby players (mixed type of training) and 10 subjects with a sedentary life style (controls). The athletes were of national competition level and trained between 6 and 9 hours a week. They had been involved in physical exercise since 4-5 years. All groups were age matched and there were no significant differences in physiological baseline characteristics between the four groups.

All subjects gave informed consent to the protocol, approved by the local ethical committee.

\subsection{Data acquisition}

Short-term ECG signals of the subjects were recorded during 10 minutes consecutively in supine position. Analogue ECG signals were digitised with a Dataq A/D converter (Dataq DI 220PGH, 8 channels, 12 bit precision, maximal $82.9 \mathrm{kHz}$ sampling rate over all channels, DATAQ Instruments Inc., Akron, OH, USA) at a rate of $1000 \mathrm{~Hz}$, providing a time resolution of $1 \mathrm{msec}$ 


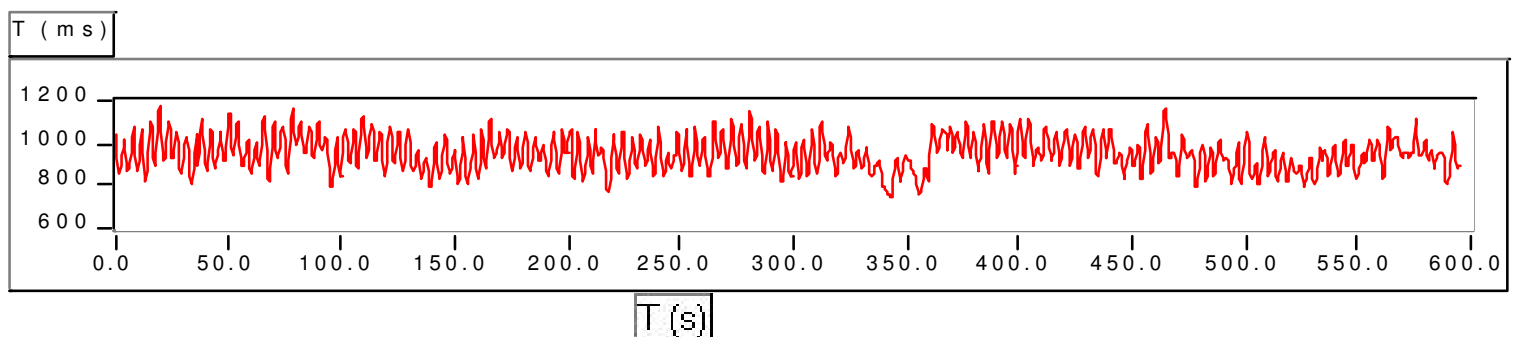

Figure 1. Tachogram obtained from an aerobic athlete.

to allow accurate RR peak detection. Real time digital signal acquisition was performed by a PC (DELL, PII $233 \mathrm{MHz}$ ) equipped with Windaq Recording Software (DATAQ Instruments Inc., Akron, OH, USA). Peak detection was performed using a self-developed LabVIEW program [13] (Laboratory Virtual Instrument Engineering Workbench version 5.0, National Instruments, Austin, TX, USA). Adequacy of peak detection was controlled by the operator before further processing of the tachogram time series

\subsection{Data analysis}

Measurements of HRV in both time and frequency domains were calculated following international standards. In the time domain, measurements included mean NN and SDNN, rMSSD and pNN50 [2]. The power spectral density of HRV was derived from the tachogram after resampling at $2 \mathrm{~Hz}$ (in order to obtain equidistant points) and FFT computed after Hanning windowing over 256 points $(128 \mathrm{~s})$. All software has been developed in house [14] and thoroughly validated. Total power (TP), low frequency (LF, 0.04-0.15 Hz) and high frequency (HF, 0.15-0.4 Hz) spectral power density were computed, as well as LF/HF.

\subsection{Non-linear dynamics methods}

The following non-linear methods were computed: fractal dimension, 1/f slope, ApEn, and Lyapunov exponent.

Non-linear (deterministic) chaos refers to a constrained kind of randomness, which may be associated with fractal geometry. A fractal has as an essential characteristic that its details at a certain scale are similar (though not necessarily identical) to those of the structure seen at larger or smaller scales.

Entropy refers to system randomness, regularity and predictability and allows systems to be classified by rate of information loss or generation.

The trajectories of a chaotic signal in phase space follow typical patterns. Closely spaced trajectories converge and diverge exponentially relative to each other. Lyapunov exponents measure the average rate of convergence/divergence of these neighboring trajectories. A positive Lyapunov exponent can be considered as a

definition of chaos provided the system is known to be deterministic.

Details on computation of all non-linear parameters were described previously $[15,16]$.

\section{Results}

A typical tachogram obtained after peak detection on a 10 min ECG recording from an aerobic trained athlete is shown in figure 1. Aerobic trained athletes showed a lower resting heart rate (also visible on figure 1) compared to controls; $50 \pm 4.6$ vs $73 \pm 14$ beats/min. Systolic blood pressure was higher in anaerobic vs controls $(147 \pm 4.1$ vs $134 \pm 16 \mathrm{mmHg})$.

Non-linear behaviour of the tachogram data sets can be shown by creating a surrogate data set. This artificial data set has exactly the same linear properties has the original (statistical characteristics (mean) and power spectral density), but has no further determinism built in. The surrogate data set is obtained as follows: compute FFT of the original, multiply phase by random phases, uniformly distributed in $[0,2 ð]$, then compute the inverse FFT [9, 17].

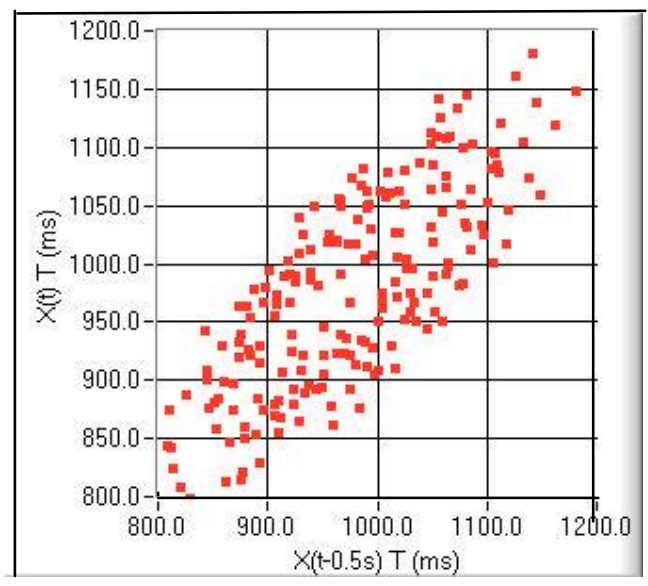

Figure 2. Phase portrait of the data shown in Fig. 1, with an embedded time delay of $0.5 \mathrm{~s}$

If the measured data are properly described by a linear process, then no significant differences from the artificial ones should be found. The original and artificial time series are compared by plotting each value against the value a certain time delay ( $\ddot{\mathrm{A}}$ ) earlier. The optimal value 
for Ät is obtained from the maximum of the autocorrelation function.

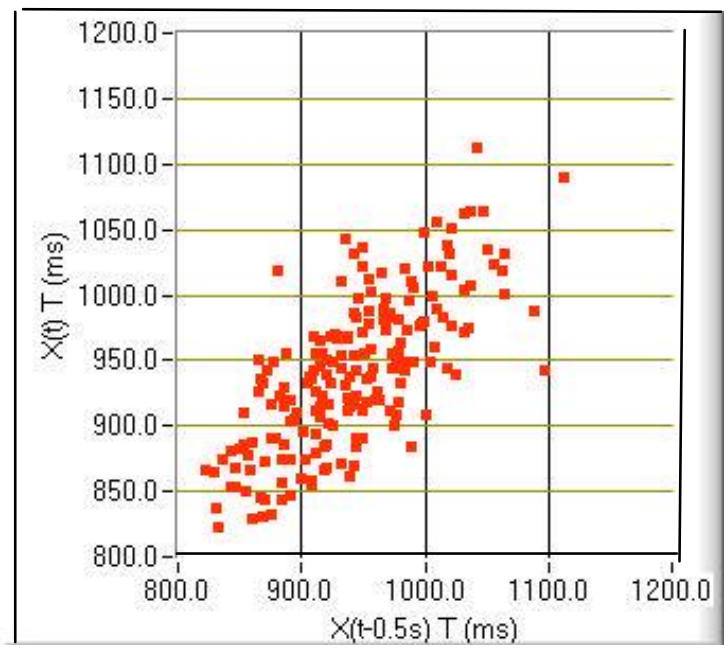

Figure 3 Phase portrait of an artificial data set consisting of the random numbers with the same linear properties as data in figure 2.

The resulting two phase portraits look qualitatively different. There is a (ellipsoidal) structure present in the original data (Fig. 2), which is not reproduced by the artificial series (Fig.3), showing non-linearity in the system.

Analysis of the frequency domain variables (Table 1), revealed that aerobic trained athletes had significantly more total power and LF and a tendency to higher HF.

Table 1. HRV parameters in the frequency domain.

\begin{tabular}{lllll}
\hline & $\begin{array}{l}\mathrm{Ln}(\mathrm{TP}) \\
\left(\mathrm{ms}^{2}\right)\end{array}$ & $\begin{array}{l}\mathrm{Ln}(\mathrm{LF}) \\
\left(\mathrm{ms}^{2}\right)\end{array}$ & $\begin{array}{l}\mathrm{Ln}(\mathrm{HF}) \\
\left(\mathrm{ms}^{2}\right)\end{array}$ & $\mathrm{LF} / \mathrm{HF}$ \\
\hline Control & $7.9 \pm 0.8$ & $6.88 \pm 0.6$ & $6.18 \pm 1.01$ & $2.4 \pm 1.5$ \\
Aerobic & $8.76 \pm 0.36^{*}$ & $7.73 \pm 0.49^{*}$ & $7.08 \pm 0.68$ & $2.15 \pm 1.7$ \\
Anaerobic & $7.82 \pm 0.56$ & $6.85 \pm 0.51$ & $6.07 \pm 0.73$ & $2.34 \pm 1.0$ \\
Rugby & $7.51 \pm 1.11$ & $6.80 \pm 1.10$ & $5.38 \pm 1.63$ & $5.0 \pm 3.2^{*}$ \\
\hline
\end{tabular}

Values are mean $\pm \mathrm{SD}, \mathrm{TP}, \mathrm{LF}$ and $\mathrm{HF}$ are $\ln$ transformed to normalize distribution.

Rugby players had a shifted sympatho-vagal balance towards larger LF power. Anaerobic athletes were not diversified from controls.

Non-linear analysis parameters are listed in Table 2. No significant differences could be shown between the different groups for any of the parameters. There is an overall tendency for parameters from the aerobic athletes group, to be somewhat smaller. Correlations between all parameters (frequency domain and non-linear) only showed a significant relationship between total power and ApEn and LF and ApEn. Among the different non-

Table 2 Parameters obtained from non-linear methods. The column of $1 / \mathrm{f}$ shows the exponent of the numerator.

\begin{tabular}{lcccc}
\hline & FD & $1 / \mathrm{f}$ & ApEn & Lyapunov \\
\hline Control & $1.27 \pm 0.08$ & $0.8 \pm 0.26$ & $1.09 \pm 0.19$ & $0.78 \pm 0.18$ \\
Aerobic & $1.24 \pm 0.26$ & $0.72 \pm 0.33$ & $0.93 \pm 0.33$ & $0.65 \pm 0.21$ \\
Anaerobic & $1.28 \pm 0.28$ & $0.93 \pm 0.42$ & $1.17 \pm 0.36$ & $0.87 \pm 0.23$ \\
Rugby & $1.24 \pm 0.29$ & $0.89 \pm 0.44$ & $0.98 \pm 0.31$ & $0.80 \pm 0.22$ \\
\hline
\end{tabular}

linear parameters, only FD and ApEn were correlated. Values of the reconstruction delay in the computation of the Lyapunov exponent varied from 3 through 11 and were calculated separately for each time series. The embedding dimension was equal to 2 . Values from the anaerobic group showed a tendency towards higher values compared to the control group.

\section{Discussion}

Linear methods interpret all regular structure in a data set as linear correlations, meaning that the intrinsic dynamics of the system are governed by the linear paradigm that small causes lead to small effects. All irregular behaviour has to be attributed to some random external input to the system. On the other hand, nonlinear, chaotic systems can produce very irregular data with purely deterministic equations of motion.

In order to show that the tachogram time series (Fig. 1) has a non-linear behaviour, we compared the original with an artificial data set. The resulting phase portraits (Fig. 2 and 3) look qualitatively different. Since both series have the same linear properties, the difference is most likely due to non-linearity in the system.

\subsection{Fractal dimension and $1 / \mathbf{f}$}

The details of a fractal at a certain scale are similar to those of the structure seen at larger or smaller scales i.e. when the heart rate of a healthy subject is recorded for 5 , 50 and $500 \mathrm{~min}$, the fast erratic fluctuations in the tachogram seem to vary in a similar manner to the slower fluctuations (self similarity at different time scales).

The scaling property is related to the $1 / \mathrm{f}$ behaviour. The different groups were not differentiated for this parameter and the exponent was close to one (Table 2).

The familiar concept of dimensions in daily life (Euclidean space) is clear: a point has the dimension 0, a line or curve has dimension 1 , a surface 2 and a volume 3. A fractal line however (achogram) spills over into two-dimensional space and therefore has a non-integer dimension between 1 and 2 . Values found between different groups are all within the same range, indicating a non-discriminatory property of this parameter. 


\subsection{Entropy}

The classification of dynamical systems via entropy stems from theoretical work of Kolmogorov in the 50's. Another mathematical approach called 'approximate entropy' (ApEn) has been introduced as quantification of regularity in data [18]. ApEn measures the likelihood that runs of patterns that are closely similar will remain close for the subsequent incremental comparison. The probabilistic nature of this parameter implies that it will be most useful to uncover subtle abnormalities or alterations in long-term data that are not otherwise apparent. Values in athletes (Table 2) are comparable to previous published work [19].

\subsection{Lyapunov exponents}

The Lyapunov exponent is a quantitative measure of separation of trajectories in phase space that diverge from their initial close positions. The magnitude of the exponent is also related to how chaotic a system is. The larger the exponent, the more chaotic the system. A periodic signal for example will have exponent zero. A positive Lyapunov exponent indicates sensitive dependenence on the initial conditions and is a diagnostic of chaos, provided the system is known to be deterministic. Results indicate the aerobic athletes to be the least chaotic.

It is important for the computation of the Lyapunov exponent to determine the optimal time delay. Some authors use a fixed value, while others prefer to calculate a time lag for each subject, which we also preferred. Values deviating too much from the optimal lag will lead to erroneous values of the Lyapunov exponents.

We conclude that: 1. Non-linear dynamics methods are more complex from the structural point of view as well as from computational; 2. Some of the non-linear methods (Lyapunov especially) may require longer data recordings, 3. Approximate entropy, based on the detection of similarity patterns in a time series, seems the most powerful of the non-linear methods used. Nonlinear methods are also graphically less appealing. Finally non-linear time series analysis is much less understood (for basic physiologic understanding) than classical methods for linear processes.

\section{Acknowledgements}

This study was supported by a grant of the Flemish Institute for the advancement of scientific and technologic research in the Industry (IWT).

\section{References}

[1] Malik M and Camm AJ, eds. Heart rate variability. Futura Publ, Armonk, NY, 1995.

[2] Aubert AE and Ramaekers D. Neurocardiology: the benefits of irregularity. The basics of methodology, physiology and current clinical applications. Acta Cardiol 1999;54:107-120.

[3] Akselrod S, Gordon D, Ubel FA, Shannon DC, Barger AC, Cohen R. Power spectral analysis of heart rate fluctuations: a quantitative probe of beat-to-beat cardiovascular control. Science 1981;213:220-222.

[4] Mortara A, La Rovere MT, Pinna GD, Prpa A, Maestri R, Febo O, Pozzollo M, Opasich C, Tavazzi L. Arterial baroreflex modulation of heart rate in chronic heart failure. Circulation 1997;96(10):3450-3458.

[5] Hartikainen JEK, Tahvanainen KUO, Kuusela TA. Shortterm measurement of heart rate variability, p. 149-176, In: Clinical guide to cardiac autonomic tests, M Malik ed., Kluwer Ac Publ, Dordrecht 1998.

[6] Katz MJ. Fractals and the analysis of waveforms. Comput Biol Med 1988;18(3):145-156.

[7] Rosenstein M, Collins JJ, De Luca CJ. A practical method for calculating largest Lyapunov exponents from small data sets. Physica D 1993;65:117-134.

[8] Persson PB, Wagner CD. General principles of chaotic dynamics. Cardiovasc Res 1996;31:332-341.

[9] Bogaert C, Beckers F, Ramaekers F, Aubert AE. Analysis of heart rate variability with correlation dimension method in a normal population and in heart transplant patients. Auton. Neurosci. 90: 142-147, 2001

[10] Kobayashi M, Musha T. 1/f fluctuations of heartbeat period. IEEE Trans Biomed Eng 1982;29(6):456-457.

[11] Pincus SM. Approximate entropy as a measure of system complexity. Proc Natl Acad Sci USA1991;88:2297-2301.

[12] Peng CK, Havlin S, Hausdorff JM, Mietus JE, Stanley HE, Goldberger AL. Fractal mechanisms and heart rate dynamics. J Electrocardiol 1996;28(suppl):59-64.

[13] Beckers F, Aubert AE, Ramaekers D, Ector H, Van de Werf F. ACTS: Automatic calculation of tachograms and systograms. Progr Biomed Res 1999;4:160-165.

[14] Aubert AE, Ramaekers D, Beckers F, Breem R, Denef C, Van de Werf F, Ector $\mathrm{H}$. The analysis of heart rate variability in unrestrained rats. Validation of methods and results. Comp Meth Progr Biomed 1999;60:197-213.

]15] Beckers F, Ramaekers D, Aubert AE. Non-linear dynamics in heart rate variability. IEEE Computers in Cardiology 2000,. 27: 131-134.

[16] Beckers F, Ramaekers D, Aubert AE. Approximate entropy of heart rate variability: validation of methods and application in heart failure. Cardiovasc Eng 2002. In press.

[17] Kantz H, Schreiber T. Nonlinear time series analysis. Cambridge, University Press, 1997

[18] Pincus SM. Approximate entropy as a measure of system complexity. Proc Natl Acad Sci 1991, 88: 2297-2301

[19] Sapoznikov D, Luria MH, Gotsman MS. Detection of regularities in heart rate variations by linear and non-linear analysis: power spectrum versus approximate entropy. Comp Meth Progr Biomed. 1995, 48: 201-209

Address for correspondence:

André E Aubert.

UZ Gasthuisberg O-N

Labo Exp Cardiology

Herestr 49

3000 Leuven Belgium

Andre.Aubert@med.kuleuven.ac.be 